Document downloaded from:

http://hdl.handle.net/10251/50012

This paper must be cited as:

González Sorribes, A.; Sala Piqueras, A.; Sanchis, R. (2013). LK stability analysis of predictor-based controllers for discrete-time systems with time-varying actuator delay. Systems and Control Letters. 62(9):764-769. doi:10.1016/j.sysconle.2013.05.009.

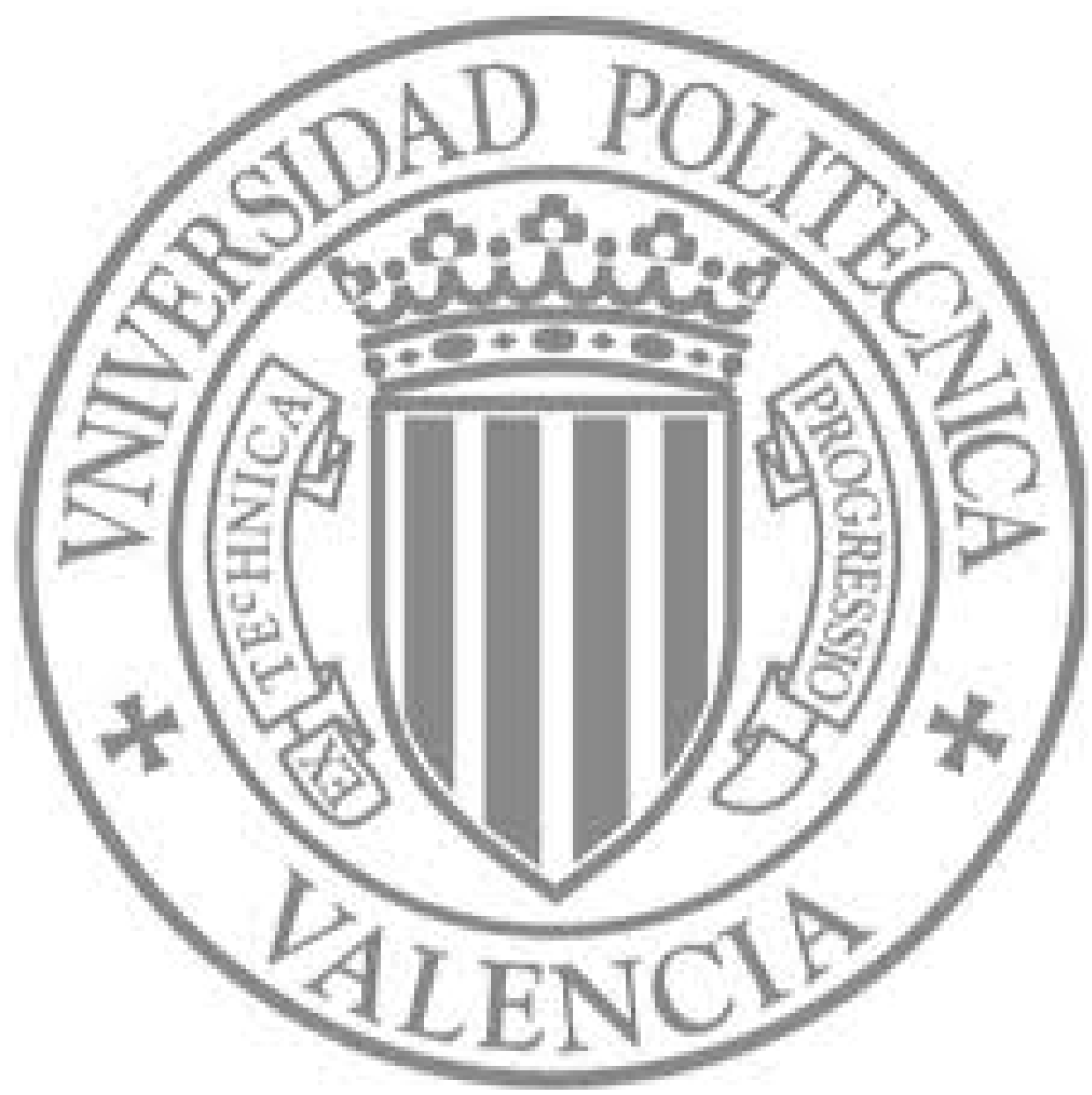

The final publication is available at

http://dx.doi.org/10.1016/j.sysconle.2013.05.009

Copyright Elsevier 


\title{
LK stability analysis of predictor-based controllers for discrete-time systems with time-varying actuator delay
}

\author{
A. Gonzalez ${ }^{\mathrm{a}, *}$, A. Sala ${ }^{\mathrm{a}}$, R. Sanchis ${ }^{\mathrm{b}}$ \\ ${ }^{a}$ Instituto Automática e Informática Industrial, \\ Universitat Politècnica de València, E-46022 Valencia, Spain \\ ${ }^{b}$ Dept. Industrial Systems Engineering, \\ Universitat Jaume I, Av. de Vicent Sos Baynat, s/n, 12071 Castellón, Spain
}

\begin{abstract}
This paper extends the scaled-small gain proposal in [1], modifying the Lyapunov-Krasovskii functional to analyze the robust stability of predictorbased state-feedback controllers for discrete-time systems with model uncertainties and unknown time-varying delays in the control input. Results show that the use of predictors improves the achieved robustness margin over static state feedback in some cases, as expected, due to the extra information available to the controller.
\end{abstract}

Keywords: Input-output approach; Discrete h-step ahead predictor; Linear matrix inequality (LMI); Lyapunov-Krasovskii (LK) functional.

\section{Introduction}

Dead-time compensation (DTC) techniques are frequently used in the control of time-delay systems (Smith predictor [2, 3, 4], Finite Spectrum Assignment [5, 6] FSA, nonlinear systems [7, 8]). Most of such schemes are mainly based on embedding a particular delay-free design into a delayed loop in such a way that stability can be guaranteed if the actual delay is equal to the nominal one used in the design phase. The issue of propagation of modeling errors may seem to be a possible drawback for DTC for large delays; hence, computing robustness margins in these cases is also a problem of practical interest.

\footnotetext{
${ }^{*}$ Corresponding author

${ }^{1}$ Email address: angonsor@upvnet.upv.es (A. Gonzalez)
} 
This paper considers the discrete-time version of the FSA $[9,10]$ to compensate actuator delays in linear systems. The paper [11] shows that, at least in some cases, the introduction of FSA-like predictors improves robust stability margins over some static state-feedback control solutions in the literature due to the extra information (past inputs) available to compute control actions. However, [11] uses a delay-free augmented realization (as done in $[12,10]$ ) whose number of decision variables and linear matrix inequality (LMI) size sharply increase with the delay bounds. On the other hand, Lyapunov-Krasovskii (LK) approaches pose LMIs whose complexity is, basically, independent of the delay [13]; for instance, predictor-based loops are analyzed with LK functionals in [14], albeit only for systems without uncertainty.

The present work complements the predictor-based analysis in $[11,14]$ by adapting the results in [1] to the stability analysis of predictor-based loops with both uncertain plant models and time-varying delays. In particular, the LK functional in [1] is modified to take into account some specific terms appearing in the predictor loops.

\section{Problem Formulation}

Let us consider the following uncertain linear discrete-time system with time-varying input delay (with known delay bounds $d_{m}, d_{M}, \tau=d_{M}-d_{m}$ ) and invertible $A$ (i.e., no state delay):

$$
\begin{aligned}
x_{k+1} & =\left(A+\Delta_{A_{k}}\right) x_{k}+\left(B+\Delta_{B_{k}}\right) u_{k-d_{k}} \quad \forall k \geq 0 \\
d_{m} & \leq d_{k} \leq d_{M} \\
x_{0} & =\eta_{x} \quad u_{\kappa}=\eta_{u}(\kappa), \quad-d_{M} \leq \kappa<0
\end{aligned}
$$

where $\eta_{x}$ is an initial condition for state and $\eta_{u}(\kappa)$ represents initial conditions for the control action input $u_{k}$. Model uncertainties $\Delta_{A_{k}}, \Delta_{B_{k}}$ are assumed to have the widely-used structure [15]:

$$
\left(\begin{array}{cc}
\Delta_{A_{k}} & \Delta_{B_{k}}
\end{array}\right)=\alpha G \Delta_{k}\left(\begin{array}{ll}
H_{A} & H_{B}
\end{array}\right)
$$

being $G, H_{A}, H_{B}$ known matrices of appropriate dimensions; $\Delta_{k}$ is a timevarying matrix of unknown elements, that satisfies the unit norm-bound inequality $\Delta_{k}^{T} \Delta_{k} \leq I$, and $\alpha$ is a positive scalar that determines the size of uncertainties (robustness margin), if so wished. 
Let us consider the predictor-based control law $[9,11]$ (discrete-time version of well-known continuous-time schemes [5]) based on Artstein's model reduction [16]:

$$
u_{k}=K z_{k} \quad z_{k}=x_{k}+\sum_{i=0}^{h-1} A^{-i-1} B u_{k-h+i}
$$

where $z_{k}$ is the transformed state $^{2}$, and $h \geq 0$ is a user-defined prediction horizon. The abuse of notation $\sum_{i=p}^{q} \Xi_{i}=0$ for the case with index bounds $q<p$ is implicitly considered above and in later developments, where $\Xi_{i}$ is any arbitrary matrix expression.

Then, the closed loop realization of system (1)-(3) is obtained from the lemma below:

Lemma 1 ([11]) For $h \geq 0$, the closed loop realization of the system (1) with the control law defined in (3) can be expressed as:

$$
\begin{aligned}
z_{k+1} & =\tilde{A}_{k} z_{k}+B_{d, k} z_{k-d_{k}}-B K z_{k-h}+\sum_{i=0}^{h-1} B_{i, k} z_{k-h+i} \\
\tilde{A}_{k} & =A+A^{-h} B K+\Delta_{A_{k}} \quad B_{d, k}=B K+\Delta_{B_{k}} K \\
B_{i, k} & =-\Delta_{A_{k}} A^{-i-1} B K
\end{aligned}
$$

The objective of this work is determining the robustness of such predictionbased strategies with a tractable methodology for long delays (instead of the delay-free augmented realizations proposed in [11], whose matrix sizes and number of decision variables sharply increase with the delay, as seen later).

Note that the sum of terms involving $B_{i, k}$ in (4) models the "accumulation" of modelling error for long predictor horizons $h$ due to $\Delta_{A_{k}}$. So, for long delays, there will be a trade-off between the delay-cancellation benefits trying to reach $h \approx d_{k}$ (i.e., trying to cancel-off the $B K$ term in $B_{d, k}$ ) and the modeling error accumulation in the prediction due to adding up $B_{i, k}$ in

\footnotetext{
${ }^{2}$ The term "prediction" is mentioned because the transformed state is obtained from the well-known convolution formula for the nominal $h$-step ahead prediction $\bar{x}_{k+h}=A^{h} x_{k}+$ $\sum_{i=0}^{h-1} A^{h-i-1} B u_{k-h+i}$, and the change of variable $z_{k}=A^{-h} \bar{x}_{k+h}$. The motivation of these controllers [16] lies in the well-known fact that, under no uncertainty and $d_{k}=h$, a controller $\bar{K}$ such that $A+B \bar{K}$ is stable allows to stabilize the delayed system (1) with control law $u_{k}=\bar{K} \bar{x}_{k+h}$, i.e.,(3) for $K=\bar{K} A^{h}$,easily shown from (4)
} 
the right-most summation in (4). This will give rise to an "optimal" value of the prediction horizon (see example section) for maximum robustness.

\section{Main Result}

In this section, a theorem generalizing state-feedback results in [1] to the above-presented predictor-based control is proposed. State-feedback will be a particular case $h=0$, and a more flexible Lyapunov-Krasovskii functional is proposed, modifying $V_{3}$ and introducing $V_{4}$ in (10) with respect to the original reference in order to reduce conservativeness. A detailed discussion is later provided in Section 3.1.

Before stating the main result, for the case $h>1$, consider an auxiliary partitioning of the range from 0 to $h-1$ in $p$ segments (being $p \in \mathbb{N}$ any choice such that $1 \leq p \leq h-1$ ) by arbitrarily choosing $p-1$ integers (denoted as $h_{1}, \ldots, h_{p-1}$ for $p>1$ ) such that $h_{0}=0, h_{p}=h-1, \quad h_{0} \leq h_{1} \leq \cdots \leq$ $h_{p-1} \leq h_{p}$ and define $\delta_{q}=h_{q}-h_{q-1}$ for $q=1, \ldots, p$.

Let us also define the following auxiliary matrices (later used in Theorem 1):

$$
\begin{aligned}
\Psi_{2} & =\left(\begin{array}{lllllll}
A+A^{h} B K & \frac{1}{2} B K & \frac{1}{2} B K & -B K & \frac{\tau}{2} B K & G
\end{array}\right) \\
\Psi_{3} & =\left(\begin{array}{lllllll}
A+A^{h} B K-I & \frac{1}{2} B K & \frac{1}{2} B K & -B K & \frac{\tau}{2} B K & G
\end{array}\right) \\
\Psi_{4} & =\left(\begin{array}{llllll}
H_{A} & \frac{1}{2} H_{B} K & \frac{1}{2} H_{B} K & -H_{A} A^{-1} B K & \frac{\tau}{2} H_{B} K & 0
\end{array}\right)
\end{aligned}
$$

and some scalars coefficients $\theta_{i}$ (later used in the Lyapunov-Krasovskii function) and $\rho_{i}, i=1,2,3$, fixed a priori, depending on the choice of the predictor horizon value $h$, defined as:

$$
\begin{gathered}
\begin{cases}0<h \leq d_{m} & \theta_{1}=h, \quad \theta_{2}=d_{m}, \quad \theta_{3}=d_{M} \\
d_{m}<h \leq d_{M} & \theta_{1}=d_{m}, \quad \theta_{2}=h, \quad \theta_{3}=d_{M} \\
h>d_{M} & \theta_{1}=d_{m}, \quad \theta_{2}=d_{M}, \quad \theta_{3}=h\end{cases} \\
\rho_{1}=\theta_{1}, \quad \rho_{2}=\theta_{2}-\theta_{1}, \quad \rho_{3}=\theta_{3}-\theta_{2}
\end{gathered}
$$

Theorem 1 For $h \geq 1$, system (4) is robustly asymptotically stable if there exists symmetric matrices $P, Q_{1}, Q_{2}, Q_{3}, R_{1}, R_{2}, R_{3}, S>0, X_{q}, Y_{q}$, for

$q=1,2, \cdots, p$, and (only if $p \neq 1$ ) matrices $X_{r s}, Y_{r s}$ for $r=1, \cdots, p-1$, and $s=r+1, \cdots, p$, and some scalar $\sigma>0$ such that the following conditions hold: 


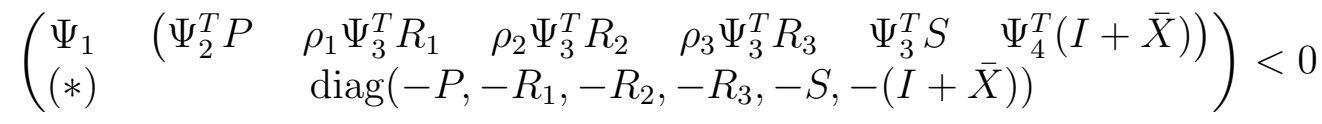

$$
\left(\begin{array}{cc}
X_{q} & I \\
I & Y_{q}
\end{array}\right)>0, \quad\left(\begin{array}{cc}
X_{r s} & -I \\
-I & Y_{r s}
\end{array}\right)>0 \quad(p \neq 1)
$$

where:

$$
\begin{gathered}
\Psi_{1}=\left(\begin{array}{llllll}
\psi_{11} & \psi_{12} & 0 & \psi_{14} & 0 & 0 \\
(*) & \psi_{22} & \psi_{23} & \psi_{24} & 0 & 0 \\
(*) & (*) & \psi_{33} & \psi_{34} & 0 & 0 \\
(*) & (*) & (*) & \psi_{44} & 0 & 0 \\
(*) & (*) & (*) & (*) & -S & 0 \\
(*) & (*) & (*) & (*) & (*) & -\sigma I
\end{array}\right), \quad \bar{X}=\sum_{q=1}^{p} X_{q} \\
\psi_{11}=-P+Q_{1}+Q_{2}+Q_{3}-R_{1}+\sum_{q=1}^{p} M_{q} \\
M_{q}=\delta_{q} K^{T} B^{T}\left\{\sum_{i=1+h_{q-1}}^{h_{q}}\left(A^{-i-1}\right)^{T} H_{A}^{T}\left(I+\bar{Y}_{q}\right) H_{A} A^{-i-1}\right\} B K \\
\bar{Y}_{q}=Y_{q}+\sum_{s=q+1}^{p} X_{q s}+\sum_{r=1}^{q-1} Y_{r q} \\
\psi_{12}=\left\{\begin{array}{ll}
0 & h \leq d_{m}, \\
R_{1} & h>d_{m}
\end{array} \quad \psi_{14}= \begin{cases}R_{1} & h \leq d_{m} \\
0 & h>d_{m}\end{cases} \right. \\
\psi_{22}=-Q_{1}-R_{2}- \begin{cases}R_{3} & h \leq d_{m} \\
R_{1} & h>d_{m}\end{cases}
\end{gathered}
$$




$$
\begin{aligned}
& \psi_{23}=\left\{\begin{array}{ll}
R_{3} & h \leq d_{m} \\
0 & d_{m}<h \leq d_{M}, \\
R_{2} & h>d_{M}
\end{array} \quad \psi_{24}= \begin{cases}R_{2} & h \leq d_{M} \\
0 & h>d_{M}\end{cases} \right. \\
& \psi_{33}=-Q_{2}-R_{3}-\left\{\begin{array}{ll}
0 & h \leq d_{M} \\
R_{2} & h>d_{M}
\end{array}, \quad \psi_{34}= \begin{cases}0 & h \leq d_{m} \\
R_{3} & h>d_{m}\end{cases} \right. \\
& \psi_{44}=-Q_{3}- \begin{cases}R_{2}+R_{1} & h \leq d_{m} \\
R_{2}+R_{3} & d_{m}<h \leq d_{M} \\
R_{3} & h>d_{M}\end{cases}
\end{aligned}
$$

The upper bound for model uncertainties can be computed by minimizing $\sigma$ subject to the above constraints. If feasibility is achieved, the proven size of tolerated model uncertainties (maximum $\alpha$ ) while keeping stability can be obtained as $\bar{\alpha}=\sqrt{1 / \sigma}$.

Proof:Let us consider the system formed by the feedback interconnection of the two subsystems $\mathcal{G}_{s}$ and $\Delta$, described below in (8) and (9) respectively, given by:

$$
\begin{aligned}
& {\left[\begin{array}{c}
z_{k+1} \\
\hline \nu_{k} \\
y_{\Sigma_{k}}
\end{array}\right]=\underbrace{\left[\begin{array}{c|cc}
\Gamma_{1} & \frac{\tau}{2} B K & \alpha G \\
\hline \Gamma_{2} & \frac{\tau}{2} B K & \alpha G \\
\Gamma_{3} & \frac{\tau}{2} H_{B} K & 0
\end{array}\right]}_{\mathcal{G}_{s}}\left[\begin{array}{c}
\bar{z}_{k} \\
\hline w_{d_{k}} \\
w_{\Sigma_{k}}
\end{array}\right]} \\
& {\left[\begin{array}{c}
w_{d_{k}} \\
w_{\Sigma_{k}}
\end{array}\right]=\underbrace{\left(\begin{array}{cc}
\Delta_{d_{k}} & 0 \\
0 & \Delta_{k}
\end{array}\right)}_{\Delta}\left[\begin{array}{c}
\nu_{k} \\
y_{\Sigma_{k}}
\end{array}\right]} \\
& \Gamma_{1}=\left(\begin{array}{lllll}
A+A^{h} B K & \frac{1}{2} B K & \frac{1}{2} B K & -B K & 0
\end{array}\right) \\
& \Gamma_{2}=\left(\begin{array}{lllll}
A+A^{h} B K-I & \frac{1}{2} B K & \frac{1}{2} B K & -B K & 0
\end{array}\right) \\
& \Gamma_{3}=\left(\begin{array}{lllll}
H_{A} & \frac{1}{2} H_{B} K & \frac{1}{2} H_{B} K & -H_{A} A^{-1} B K & -H_{A}
\end{array}\right) \\
& \bar{z}_{k}=\left(\begin{array}{lllll}
z_{k}^{T} & z_{k-d_{m}}^{T} & z_{k-d_{M}}^{T} & z_{k-h}^{T} & \phi_{k}^{T}
\end{array}\right)^{T}
\end{aligned}
$$

where $\phi_{k}=\sum_{i=1}^{h-1} A^{-i-1} B K z_{k-h+i}$ and, from the definition of $\Gamma_{2}$, we have $\nu_{k}=z_{k+1}-z_{k}$. Also, $w_{d_{k}}$ is defined as:

$$
w_{d_{k}}=\frac{2}{\tau}\left[z_{k-d_{k}}-\frac{1}{2}\left(z_{k-d_{M}}+z_{k-d_{m}}\right)\right]
$$


and satisfies [1, Lemma 2], i.e., $w_{d_{k}}=\Delta_{d_{k}} \nu_{k}$, with $\left\|\Delta_{d_{k}}\right\|_{\infty} \leq 1$. On the other hand, the model uncertainties have been pulled out by defining the following input $w_{\Sigma_{k}}$ to $\mathcal{G}_{s}$ :

$$
\begin{aligned}
w_{\Sigma_{k}}= & \Delta_{k} y_{\Sigma_{k}} \\
y_{\Sigma_{k}}= & H_{A} z_{k}+\frac{1}{2} H_{B} K\left(z_{k-d_{m}}+z_{k-d_{M}}\right) \\
& -H_{A} A^{-1} B K z_{k-h}+\frac{\tau}{2} H_{B} K w_{d_{k}}-H_{A} \phi_{k}
\end{aligned}
$$

It can be proved that (8) and (9) are equivalent to (4), following an analogous development to that in [1, Proposition 1].

Then, consider the following discrete-time Lyapunov-Krasovskii functional candidate:

$$
V(k)=V_{1}(k)+V_{2}(k)+V_{3}(k)+\sum_{q=1}^{p} V_{4}(k, q)
$$

with

$$
\begin{aligned}
V_{1}(k) & =z_{k}^{T} P z_{k}, \quad V_{2}(k)=\sum_{i=k-d_{m}}^{k-1} z_{i}^{T} Q_{1} z_{i}+\sum_{i=k-d_{M}}^{k-1} z_{i}^{T} Q_{2} z_{i}+\sum_{i=k-h}^{k-1} z_{i}^{T} Q_{3} z_{i} \\
V_{3}(k) & =\rho_{1} \sum_{i=-\theta_{1}} \sum_{j=k+i}^{k-1} \nu_{j}^{T} R_{1} \nu_{j}+\rho_{2} \sum_{i=-\theta_{2}}^{-\theta_{1}-1} \sum_{j=k+i}^{k-1} \nu_{j}^{T} R_{2} \nu_{j}+\rho_{3} \sum_{i=-\theta_{3}}^{\theta_{2}-1} \sum_{j=k+i}^{k-1} \nu_{j}^{T} R_{3} \nu_{j} \\
V_{4}(k, q) & =\delta_{q} \sum_{i=1+h_{q-1}}^{h_{q}} \sum_{j=k-h+i}^{k-1} z_{j}^{T} K^{T} B^{T}\left(A^{-i-1}\right)^{T} H_{A}^{T}\left(I+\bar{Y}_{q}\right) H_{A} A^{-i-1} B K z_{j}
\end{aligned}
$$

being $\theta_{i}$ and $\rho_{i}(\mathrm{i}=1,2,3)$ defined in $(5)$. Let us introduce the following auxiliary notation:

$$
\begin{aligned}
\hat{\Psi}_{2} & =\left(\begin{array}{llllll}
A+A^{h} B K & \frac{1}{2} B K & \frac{1}{2} B K & -B K & \frac{\tau}{2} B K & \alpha G
\end{array}\right) \\
\hat{\Psi}_{3} & =\left(\begin{array}{llllll}
A+A^{h} B K-I & \frac{1}{2} B K & \frac{1}{2} B K & -B K & \frac{\tau}{2} B K & \alpha G
\end{array}\right) \\
R & =\rho_{1}^{2} R_{1}+\rho_{2}^{2} R_{2}+\rho_{3}^{2} R_{3} \\
\bar{z}_{k}^{\prime} & =\left(\begin{array}{llllll}
z_{k}^{T} & z_{k-d_{m}}^{T} & z_{k-d_{M}}^{T} & z_{k-h}^{T} & w_{d_{k}}^{T} & w_{\Sigma_{k}}^{T}
\end{array}\right)^{T} \\
\bar{z}_{k}^{a} & =\left(\begin{array}{lllll}
z_{k}^{T} & z_{k-d_{m}}^{T} & z_{k-d_{M}}^{T} & z_{k-h}^{T}
\end{array}\right)^{T}
\end{aligned}
$$




$$
\begin{aligned}
& \overline{\mathcal{Q}}=\left(\begin{array}{cccc}
Q_{1}+Q_{2}+Q_{3} & 0 & 0 & 0 \\
0 & -Q_{1} & 0 & 0 \\
0 & 0 & -Q_{2} & 0 \\
0 & 0 & 0 & -Q_{3}
\end{array}\right) \\
& \overline{\mathcal{R}}=\left(\begin{array}{cccc}
\hat{\psi}_{11} & \hat{\psi}_{12} & 0 & \hat{\psi}_{14} \\
(*) & \hat{\psi}_{22} & \hat{\psi}_{23} & \hat{\psi}_{24} \\
(*) & (*) & \hat{\psi}_{33} & \hat{\psi}_{34} \\
(*) & (*) & (*) & \hat{\psi}_{44}
\end{array}\right) \\
& \hat{\psi}_{11}=-R_{1}, \quad \hat{\psi}_{12}=\left\{\begin{array}{ll}
0 & h \leq d_{m} \\
R_{1} & h>d_{m}
\end{array}, \quad \hat{\psi}_{14}= \begin{cases}R_{1} & h \leq d_{m} \\
0 & h>d_{m}\end{cases} \right. \\
& \hat{\psi}_{22}=-R_{2}-\left\{\begin{array}{ll}
R_{3} & h \leq d_{m} \\
R_{1} & h>d_{m}
\end{array}, \quad \hat{\psi}_{23}= \begin{cases}R_{3} & h \leq d_{m} \\
0 & d_{m}<h \leq d_{M} \\
R_{2} & h>d_{M}\end{cases} \right. \\
& \hat{\psi}_{24}=\left\{\begin{array}{ll}
R_{2} & h \leq d_{M} \\
0 & h>d_{M}
\end{array}, \quad \hat{\psi}_{33}=-R_{3}- \begin{cases}0 & h \leq d_{M} \\
R_{2} & h>d_{M}\end{cases} \right. \\
& \hat{\psi}_{34}=\left\{\begin{array}{ll}
0 & h \leq d_{m} \\
R_{3} & h>d_{m}
\end{array}, \quad \hat{\psi}_{44}=- \begin{cases}R_{2}+R_{1} & h \leq d_{m} \\
R_{2}+R_{3} & d_{m}<h \leq d_{M} \\
R_{3} & h>d_{M}\end{cases} \right.
\end{aligned}
$$

With the above notation, the forward difference $\Delta V(k)=V(k+1)-V(k)$ for $k \geq d_{M}$ can be expressed as:

$$
\Delta V(k)=\Delta V_{1}(k)+\Delta V_{2}(k)+\Delta V_{3}(k)+\sum_{q=1}^{p} \Delta V_{4}(k, q)
$$

with

$$
\Delta V_{1}(k)=\bar{z}_{k}^{\prime T}\left\{\hat{\Psi}_{2}^{T} P \hat{\Psi}_{2}\right\} \bar{z}_{k}^{\prime}-z_{k}^{T} P z_{k}, \quad \Delta V_{2}(k)=\bar{z}_{k}^{a^{T}} \overline{\mathcal{Q}} \bar{z}_{k}^{a}
$$




$$
\begin{aligned}
& \Delta V_{3}(k)=\nu_{k}^{T} R \nu_{k}-\rho_{1} \sum_{i=-\theta_{1}}^{-1} \nu_{i}^{T} R_{1} \nu_{i}-\rho_{2} \sum_{i=-\theta_{2}}^{-\theta_{1}-1} \nu_{i}^{T} R_{2} \nu_{i}-\rho_{3} \sum_{i=-\theta_{3}}^{-\theta_{2}-1} \nu_{i}^{T} R_{3} \nu_{i} \\
& \leq \nu_{k}^{T} R \nu_{k}-\left(\sum_{i=-\theta_{1}}^{-1} \nu_{i}\right)^{T} R_{1}\left(\sum_{i=-\theta_{1}}^{-1} \nu_{i}\right)-\left(\sum_{i=-\theta_{2}}^{-\theta_{1}-1} \nu_{i}\right)^{T} R_{2}\left(\sum_{i=-\theta_{2}}^{-\theta_{1}-1} \nu_{i}\right) \\
& -\left(\sum_{i=-\theta_{3}}^{-\theta_{2}-1} \nu_{i}\right)^{T} R_{3}\left(\sum_{i=-\theta_{3}}^{-\theta_{2}-1} \nu_{i}\right)=\nu_{k}^{T} R \nu_{k}-\left(z_{k}-z_{k-\theta_{1}}\right)^{T} R_{1}\left(z_{k}-z_{k-\theta_{1}}\right) \\
& -\left(z_{k-\theta_{1}}-z_{k-\theta_{2}}\right)^{T} R_{2}\left(z_{k-\theta_{1}}-z_{k-\theta_{2}}\right)-\left(z_{k-\theta_{2}}-z_{k-\theta_{3}}\right)^{T} R_{3}\left(z_{k-\theta_{2}}-z_{k-\theta_{3}}\right)
\end{aligned}
$$

hence, we can write $\Delta V_{3}(k)$ as:

$$
\Delta V_{3}(k) \leq \bar{z}_{k}^{\prime T}\left\{\hat{\Psi}_{3}^{T} R \hat{\Psi}_{3}\right\} \bar{z}_{k}^{\prime}+\bar{z}_{k}^{a^{T}} \overline{\mathcal{R}} \bar{z}_{k}^{a}
$$

and, finally

$\Delta V_{4}(k, q)=z_{k}^{T} M_{q} z_{k}-\delta_{q} \sum_{i=1+h_{q-1}}^{h_{q}} z_{k-h+i}^{T} K^{T} B^{T}\left(A^{-i-1}\right)^{T} H_{A}^{T}\left(I+\bar{Y}_{q}\right) H_{A} A^{-i-1} B K z_{k-h+i}$

Now, let us define the function $J(k)$, for some matrix $S>0$ given by:

$$
J(k)=\nu_{k}^{T} S \nu_{k}+y_{\Sigma_{k}}^{T} y_{\Sigma_{k}}-w_{d_{k}}^{T} S w_{d_{k}}-w_{\Sigma_{k}}^{T} w_{\Sigma_{k}}
$$

Then, proving $\Delta V(k)+J(k)<0$ for all $k$, decomposing $S=T^{T} T$, letting ${ }^{3}$ $T_{y}=\operatorname{diag}(T, I)$ and $T_{w}=\operatorname{diag}(T, I)$, by virtue of scaled small-gain theorem, $\left\|T_{y} o \mathcal{G}_{s} o T_{w}^{-1}\right\|_{\infty}<1$ holds and the closed loop is stable, following the same argumentation as in [1] because the infinity norm of system $\Delta$ defined in (8) is lower than 1 from the definitions of $\Delta_{d_{k}}$ and $\Delta_{k}$.

Thus, in order to express $\Delta V(k)+J(k)<0$ as an LMI, let us make the following partition in $p$ intervals over $\phi_{k}$ :

$$
\phi_{k}=\sum_{q=1}^{p} \phi_{q, k}, \quad \phi_{q, k}=\sum_{i=1+h_{q-1}}^{h_{q}} A^{-i-1} B K z_{k-h+i}
$$

\footnotetext{
${ }^{3}$ The dimensions of identity matrices in $T_{y}$ and $T_{w}$ may be different if $\Delta_{k}$ were not square.
} 
Then, $y_{\Sigma_{k}}$ can be expressed as:

$$
\begin{aligned}
y_{\Sigma_{k}} & =\Psi_{4} \bar{z}_{k}^{\prime}-H_{A} \sum_{q=1}^{p} \phi_{q, k} \\
\Psi_{4} & =\left(\begin{array}{llllll}
H_{A} & \frac{1}{2} H_{B} K & \frac{1}{2} H_{B} K & -H_{A} A^{-1} B K & \frac{\tau}{2} H_{B} K & 0
\end{array}\right)
\end{aligned}
$$

Then (denoting with $[*]$ the suitable transpose terms):

$$
\begin{aligned}
y_{\Sigma_{k}}^{T} y_{\Sigma_{k}}=\bar{z}_{k}^{\prime} \Psi_{4}^{T} \Psi_{4} \bar{z}_{k}^{\prime} & -\sum_{q=1}^{p}\left(\bar{z}_{k}^{\prime T} \Psi_{4}^{T} H_{A} \phi_{q, k}+[*]\right) \\
& +\sum_{q=1}^{p} \phi_{q, k}^{T} H_{A}^{T} H_{A} \phi_{q, k}+\sum_{q=1}^{p-1} \sum_{r=q+1}^{p}\left(\phi_{q, k}^{T} H_{A}^{T} H_{A} \phi_{r, k}+[*]\right)
\end{aligned}
$$

Applying standard cross-product Lemma, for some matrices $X_{q}, Y_{q}, X_{r s}, Y_{r s}$ satisfying (6):

$$
\begin{gathered}
-\sum_{q=1}^{p}\left(\bar{z}_{k}^{\prime T} \Psi_{4}^{T} H_{A} \phi_{q, k}+[*]\right) \leq \sum_{q=1}^{p}\left(\bar{z}_{k}^{\prime T} \Psi_{4}^{T} X_{q} \Psi_{4} \bar{z}_{k}^{\prime}+\phi_{q, k}^{T} H_{A}^{T} Y_{q} H_{A} \phi_{q, k}\right) \\
\sum_{q=1}^{p-1} \sum_{r=q+1}^{p}\left(\phi_{q, k}^{T} H_{A}^{T} H_{A} \phi_{r, k}+[*]\right) \\
\leq \sum_{q=1}^{p-1} \sum_{r=q+1}^{p}\left(\phi_{q, k}^{T} H_{A}^{T} X_{q r} H_{A} \phi_{q, k}+\phi_{r, k}^{T} H_{A}^{T} Y_{q r} H_{A} \phi_{r, k}\right)
\end{gathered}
$$

and, regrouping the terms at the right-hand side, we have:

$$
\begin{aligned}
\sum_{q=1}^{p-1} \sum_{r=q+1}^{p}\left(\phi_{q, k}^{T} H_{A}^{T} X_{q r} H_{A} \phi_{q, k}\right. & \left.+\phi_{r, k}^{T} H_{A}^{T} Y_{q r} H_{A} \phi_{r, k}\right) \\
& =\sum_{q=1}^{p} \phi_{q, k}^{T} H_{A}^{T}\left(\sum_{r=q+1}^{p} X_{q r}+\sum_{r=1}^{q-1} Y_{r q}\right) H_{A} \phi_{q, k}
\end{aligned}
$$

Hence, the term $y_{\Sigma_{k}}^{T} y_{\Sigma_{k}}$ can be bounded as:

$$
y_{\Sigma_{k}}^{T} y_{\Sigma_{k}} \leq \bar{z}_{k}^{\prime} T \Psi_{4}^{T}(I+\bar{X}) \Psi_{4} \bar{z}_{k}^{\prime}+\sum_{q=1}^{p} \phi_{q, k}^{T} H_{A}^{T}\left(I+\bar{Y}_{q}\right) H_{A} \phi_{q, k}
$$


Applying Jensen's inequality, and taking into account the definition of $\phi_{q, k}$ (Equation (14)), we have (18) below

$$
\begin{aligned}
& y_{\Sigma_{k}}^{T} y_{\Sigma_{k}} \leq \bar{z}_{k}^{\prime T} \Psi_{4}^{T}(I+\bar{X}) \Psi_{4} \bar{z}_{k}^{\prime} \\
& +\sum_{q=1}^{p} \delta_{q}\left[\sum_{i=1+h_{q-1}}^{h_{q}} z_{k-h+i}^{T} K^{T} B^{T}\left(A^{-i-1}\right)^{T} H_{A}^{T}\left(I+\bar{Y}_{q}\right) H_{A} A^{-i-1} B K z_{k-h+i}\right]
\end{aligned}
$$

Hence, using (11), (13) and (18), we have

$$
J(k)+\Delta V(k)=\bar{z}_{k}^{T}\left[\Psi_{1}+\hat{\Psi}_{2}^{T} P \hat{\Psi}_{2}+\hat{\Psi}_{3}^{T}(R+S) \hat{\Psi}_{3}+\Psi_{4}^{T}(I+\bar{X}) \Psi_{4}\right] \bar{z}_{k}^{\prime}
$$

Applying suitable algebraic manipulations, Schur complement, and congruence transformation, the proof is completed.

\subsection{Discussion}

Let us discuss the improvements over previous literature provided by the above result.

First, the advantages over [1] are:

- Although the result in Theorem 1 has been stated for $h \geq 1$, the case $h=0$ can be equally developed resulting in smaller LMIs (details omitted for brevity). Indeed, in that case, $V_{4}(k)=0$ and summation terms in the Lyapunov functional candidate (10) involving $Q_{3}$ in $V_{2}(k)$ and $R_{1}$ in $V_{3}(k)$ are equal to zero. Hence, the resulting LK functional is the same as in [1], except for some bounds in $V_{3}(k)$ (see below).

- it generalizes the referred results to predictor cases $(h \neq 0)$;

- The straightforward adaptation of $V_{3}$ in [1] including the state $z_{k-h}$ would be:

$$
V_{3}(k)=h \sum_{i=-h}^{-1} \sum_{j=k+i}^{k-1} \nu_{j}^{T} R_{1} \nu_{j}+d_{m} \sum_{i=-d_{m}}^{-1} \sum_{j=k+i}^{k-1} \nu_{j}^{T} R_{2} \nu_{j}+d_{M} \sum_{i=-d_{M}}^{-1} \sum_{j=k+i}^{k-1} \nu_{j}^{T} R_{3} \nu_{j}
$$

With this choice of $V_{3}$ there are some Jensen-inequality terms that result in some matrices multiplied by $h, d_{m}$ and $d_{M}$. However, with our proposal, the Jensen terms will be multiplied only by the differences between those parameters, see (5). 
- Last, $V_{4}$ is introduced to handle the rightmost summation in (17) avoiding conservative boundings (details omitted for brevity). Furthermore, the partition in $p$ intervals over $\phi_{k}$ also entails a possible reduction of conservativeness due to the introduction of new decision variables in LMI constraints in Theorem 6, namely $X_{q}, Y_{q}, X_{r s}, Y_{r s}$.

Regarding the improvements with respect to our previous work [11], the robustness analysis there proposes an augmented delay-free time-varying realization $\psi_{k+1}=\mathcal{A}\left(d_{k}\right) \psi_{k}+\mathcal{B} u_{k}$ where $\psi_{k}=\left(\begin{array}{llll}x_{k}^{T} & x_{k-1}^{T} \ldots x_{k-d_{M}}^{T}\end{array}\right)^{T}$. A parameter-dependent Lyapunov function $\psi_{k}^{T} P\left(d_{k}\right) \psi_{k}$ requires

$$
\frac{1}{2}\left(d_{M}-d_{m}+1\right)\left[\left(n \cdot d_{M}+n\right)^{2}+n\left(d_{M}+1\right)\right]+\left(d_{M}-d_{m}+1\right)^{2}
$$

decision variables. In the proposal here, the number of variables involved in the LMIs (1) is

$$
4 n^{2}+4 n+\frac{1}{2}\left(n_{\Delta}^{2}+n_{\Delta}\right)\left(p^{2}+p\right)
$$

where $n$ is the process order and $n_{\Delta}$ is the number of columns of the uncertainty matrix $\Delta_{k}$. Hence, we avoid the number of decision variables being quadratic in $d_{M}$ (computational resources were quickly exhausted for long delays with the approach in [11]).

Of course, the proposed LK functional in this work can be considered as a particular case of the very flexible Lyapunov function in [11] (indeed, it is obvious that a discrete-time LK functional is a quadratic form on present and past states); however, for long maximum delays the result presented here requires a much lower computational effort while keeping the robustness results reasonably close to the augmented realization approach, at least in the tested examples.

\section{Numerical examples}

Example 1 Consider the system (1) with:

$$
\begin{aligned}
A & =\left(\begin{array}{cc}
0.8 & 0 \\
0 & 0.9
\end{array}\right), \quad B=\left(\begin{array}{cc}
-0.1 & 0 \\
-0.1 & -0.1
\end{array}\right), \quad G=\left(\begin{array}{l}
1 \\
0
\end{array}\right) \\
H_{A} & =\left(\begin{array}{ll}
1 & 0
\end{array}\right), \quad H_{B}=\left(\begin{array}{ll}
0 & 0
\end{array}\right)
\end{aligned}
$$

and the predictor-based control law defined in (3), that is, $u_{k}=K z_{k}$, with $K=\bar{K} A^{h}$ and $\bar{K}=I_{2 \times 2}$. Note that the closed loop equations and the 
Table 1: Proved maximum $\bar{\alpha}$ given a delay interval for static (SSF) and predictor-based (PBF) feedback

\begin{tabular}{c|ccc}
\hline \hline Delay range: & {$[1$, Th. 2(ii)] (SSF) } & Theorem 1 (PBF) & Theorem 1 (PBF), p=2 \\
\hline \hline $2 \leq d_{k} \leq 7$ & 0.1942 & $0.2349\left(h_{\text {opt }}=2\right)$ & $0.2349\left(h_{\text {opt }}=2, h_{1}=0\right.$ or 1$)$ \\
$5 \leq d_{k} \leq 10$ & 0.1541 & $0.2206\left(h_{\text {opt }}=4\right)$ & $0.2212\left(h_{\text {opt }}=4, h_{1}=2\right)$ \\
$8 \leq d_{k} \leq 15$ & 0.1032 & $0.2078\left(h_{\text {opt }}=6\right)$ & $0.2097\left(h_{\text {opt }}=7, h_{1}=3\right)$ \\
$33 \leq d_{k} \leq 38$ & infeasible & $0.1885\left(h_{\text {opt }}=10\right)$ & $0.1972\left(h_{\text {opt }}=13, h_{1}=7\right)$ \\
\hline
\end{tabular}

uncertainty description matrices are analogous to the stability analysis in [1, Example 2] for zero predictor horizon $h\left(z_{k}=x_{k}\right)$, which amounts to an static state feedback $u_{k}=\bar{K} x_{k}$. The choice $\bar{K}=I$ makes comparative analysis with the cited work possible.

Table 1 compares the maximum allowable upper bound of the robustness parameter $\bar{\alpha}$ (Equation (2)) achieved with the same gain in three settings:

1. static state feedback $u_{k}=\bar{K} x_{k}$, directly using [1, Theorem 2].

2. predictor-based controller $\left(u_{k}=K z_{k}, K=\bar{K} A^{h}\right)$, searching by Theorem 1 for the optimal value of $h$ in the range $0<h \leq d_{M}$ for maximum robustness (by testing all combinations with the result proposed in this paper), to be denoted as $h_{\text {opt }}$.

3. The same setting as the previous one but using delay partitioning $(p \neq$ 1) in (10) for decreased conservativeness.

In particular, the system has been proved robustly asymptotically stable for all $\alpha \leq \bar{\alpha}$ in (2) with given delay interval $d_{m} \leq d_{k} \leq d_{M}$ in the leftmost column of Table 1. The used software was YALMIP (03/2012), SEDUMI 1.3 and MATLAB R2012a, on an Intel Core ${ }^{T M} i 5-2450 M C P U$ at $2.50 \mathrm{GHz}$.

For comparison, in order to assess how much is traded-off in robustness bounds versus computation time with respect to the augmented delay-free realization in [11], let us consider, for instance, the case $8 \leq d_{k} \leq 15$ :

- [11, Theorem 4.2] obtains a value $\bar{\alpha}=0.2112$ with 4288 decision variables and $h_{\text {opt }}=8$ (CPU-time: 420 sec),

- Theorem $1(p=1)$ obtains $\bar{\alpha}=0.2078$ with 26 decision variables (row 3 of Table 1) (CPU-time: $1.9 \mathrm{sec})$, and 
- Theorem 1 with delay partitioning $(p=2)$ obtains $\bar{\alpha}=0.2097$ with 30 decision variables (CPU-time: $2.3 \mathrm{sec}$ ).

So, similar robustness margins are obtained in less than $1 \%$ of the computing time.

Decision variables grow too high with the delay in [11] so the case $33 \leq$ $d_{k} \leq 38$ could not be solved with the cited techniques due to limitations in available computer resources.

In summary, Table 1 shows that the use of the predictor-based controller improves the provable delay ranges, confirming the results in [11] from a different methodology with much lower computational cost for large delays.

Example 2 Let us consider a second example already studied in [17]. The system has the form described in (1) with:

$$
A=\left(\begin{array}{cc}
0.9231 & -0.0010 \\
0.0966 & 1.0100
\end{array}\right), \quad B=\left(\begin{array}{l}
0.0384 \\
0.0120
\end{array}\right)
$$

for sampling period $T_{s}=0.1 s$. The model uncertainties are given by (2) with:

$$
G=\left(\begin{array}{ll}
0.01 & 0.01
\end{array}\right), \quad H_{A}=\left(\begin{array}{l}
1 \\
1
\end{array}\right), \quad H_{B}=1
$$

In this case, the process is open-loop unstable. The control law proposed in [17] $u_{k}=\bar{K} x_{k}$ with $\bar{K}=-\left(\begin{array}{ll}1.2625 & 1.2679\end{array}\right)$ stabilizes the delay-free system.

For the case $15 \leq d_{k} \leq 18$, static state-feedback Theorem 2 (ii) [1] obtains $\alpha=0.05$. Theorem 1 in this work gets $\bar{\alpha}=1.38$ with $h_{\text {opt }}=15$ and $p=1$; so, $h_{\text {opt }}$ turns out equal to the minimum delay. For instance, tolerance to modeling error for $h=10$ reduces to 0.94 . On the other hand, taking higher values of $p, \bar{\alpha}$ can be stretched to 1.46 (same $h_{\text {opt }}$ ), and augmented realization obtains $\bar{\alpha}=1.81$.

So, at least for this case, the unstable process seems more sensitive to the accuracy of the delay prediction than to the accumulation of error due to $B_{i, k}$ in (4).

\section{Conclusion}

In this paper, a generalization of the ideas in [1] to robust stability analysis of discrete-time predictor-based control loops has been provided, as well 
as some improvements in the Lyapunov-Krasovskii functional, allowing for reduced conservativeness in stability margins in the predictor case. An inputoutput approach (scaled small-gain) has been used to derive a set of LMI con-

straints, with a number of decision variables independent of the size of the delay bounds and prediction horizon. Numerical examples have shown how the use of predictors may give better robustness for the same delay ranges compared to static state feedback, as intuitively expected.

\section{References}

[1] X. Li, H. Gao, A new model transformation of discrete-time systems with time-varying delay and its application to stability analysis, IEEE Transactions on Automatic Control 56 (2011) 2172-2178.

[2] Q. Hu, J. Xu, T. Lee, Iterative learning control design for smith predictor, Systems \& Control Letters 44 (2001) 201-210.

[3] O. Smith, Closer control of loops with dead time, Chemical Engineering Progress 53 (1959) 217-219.

[4] J. Normey-Rico, E. Camacho, Control of dead-time processes, Springer, 2007.

[5] A. Z. Manitius, A. W. Olbrot, Finite spectrum assignment problem for systems with delays, IEEE Transactions on Automatic Control 24 (1979) 541-553.

[6] Y. Fiagbedzi, Stable transformations for control variable delayed systems, Systems \& Control Letters 57 (2008) 708-713.

[7] M. Krstić, Delay compensation for nonlinear, adaptive, and PDE systems, Birkhauser, 2009.

[8] N. Bekiaris-Liberis, M. Jankovic, M. Krstic, Compensation of statedependent state delay for nonlinear systems, Systems \& Control Letters 61 (2012) 849-856.

[9] R. Lozano, P. Castillo, P. Garcia, A. Dzul, Robust prediction-based control for unstable delay systems: Application to the yaw control of a mini-helicopter, Automatica 40 (2004) 603-612. 
[10] T. Santos, D. Limon, J. Normey-Rico, T. Alamo, On the explicit deadtime compensation for robust model predictive control, Journal of Process Control 22 (2011) 236-246.

[11] A. Gonzalez, A. Sala, P. Garcia, P. Albertos, Robustness analysis of discrete predictor-based controllers for input-delay systems, International Journal of System Science 44 (2013) 232-239.

[12] L. Hetel, J. Daafouz, C. Iung, Equivalence between the LyapunovKrasovskii functionals approach for discrete delay systems and that of the stability conditions for switched systems, Nonlinear Analysis: Hybrid Systems 2 (2008) 697-705.

[13] E. Fridman, U. Shaked, Input-output approach to stability and 12-gain analysis of systems with time-varying delays, Systems \& Control Letters 55 (2006) 1041-1053.

[14] A. Gonzalez, A. Sala, P. Albertos, Predictor-based stabilization of discrete time-varying input-delay systems, Automatica 48 (2012) 454-457.

[15] $\mathrm{K} . \mathrm{Gu}, \mathrm{H}_{\infty}$ control of systems under norm bounded uncertainties in all system matrices, IEEE Transactions on Automatic Control 39 (1994) 1320-1322.

[16] Z. Artstein, Linear systems with delayed control: A reduction, IEEE Transactions on Automatic Control 27 (1982) 869-879.

[17] L. Hu, T. Bai, P. Shi, Z. Wu, Sampled-data control of networked linear control systems, Automatica 43 (2007) 903-911. 